\title{
Is surgery justified for 80 -year-old or older intracranial meningioma patients? A systematic review
}

\author{
Ilari Rautalin ${ }^{1}$ (D) $\cdot$ Mika Niemelä $^{1} \cdot$ Miikka Korja $^{1}$
}

Received: 20 December 2019 / Revised: 10 February 2020 / Accepted: 6 March 2020 / Published online: 4 April 2020

(C) The Author(s) 2020

\begin{abstract}
Since the number of elderly people with intracranial meningiomas (IM) continues to rise, surgical treatment has increasingly become a considerable treatment option, even in very old ( $\geq 80$ years old) meningioma patients. Since little is known about whether meningioma surgery in this age group is safe and justified, we conducted a systematic review to summarize the results of surgical outcomes in very old meningioma patients. We performed a systematic literature search in Pubmed, Cochrane Library, and Scopus databases. Primarily, we extracted 1-month and 1-year survival rates, and 1-year morbidity rates, as well as information about preoperative morbidity, operative complications, meningioma size, location, histology, and peritumoral edema. Quality of the included studies was evaluated by Cochrane Collaboration Handbook and Critical Appraisal Skills Program. From the 1039 reviewed articles, seven retrospective studies fulfilled our eligibility criteria. Motor deficits (27-65\%) and mental changes (51-59\%) were the most common indications for surgery. One-month and 1-year mortality rates varied between $0-23.5 \%$ and $9.4-27.3 \%$, respectively. Most of the operated IM patients (41.2-86.5\%) improved their performance during postoperative follow-up. Impaired preoperative performance and comorbidities were most commonly related to higher postoperative mortality. None of the studies fulfilled the criteria of high quality. Based on the evidence currently available, surgical treatment of very old IM patients seems to improve the performance of highly selected individuals. Given the rapid increase of the aging population, more detailed retrospective studies as well as prospective studies are needed to prove the outcome benefits of surgery in this patient group.
\end{abstract}

Keywords Elderly $\cdot$ Intracranial meningioma $\cdot$ Surgery $\cdot$ Systematic review $\cdot 80$ years old or older

\section{Introduction}

Intracranial meningioma (IM) is the most common primary intracranial tumor, accounting for over one-third of all intracranial tumors [25]. Since the growth rate of IMs is usually slow and the majority of tumors are benign ((World Health Organization (WHO) grades I-II)) [13], IMs may grow remarkably large before causing neurological or neuropsychological symptoms. As the life expectancy has rapidly increased in high-income countries [26], even octogenarians (80-90 years old) are often in relatively good condition, live

Electronic supplementary material The online version of this article (https://doi.org/10.1007/s10143-020-01282-7) contains supplementary material, which is available to authorized users.

Ilari Rautalin

ilari.rautalin@helsinki.fi

1 Department of Neurosurgery, University of Helsinki and Helsinki University Hospital, P.O. Box 266, FI-00029 Helsinki, Finland independently at home, and have close to 10 years of life ahead of them [24]. Along with female sex, elderly age is among the most important risk factors for an increased chance of developing meningiomas [25]. As such, neurosurgical meningioma operations are likely to become more common even among very old patients ( 80 years old or older).

Increasing age is known to increase the risks of operative mortality and morbidity in cranial neurosurgery [18]. Indeed, three large register-based studies $[1,2,23]$ have suggested that the operative risks for very old (i.e., $\geq 80$ years) IM patients may increase as much as 15 times higher as compared with IM patients under 80 years old. On the other hand, two previous systematic reviews of elderly ( $\geq 65$ years old) IM patients have shown that a proper patient selection process may decrease the operative risks, even in old IM patients [11, 17]. However, there is no overview for very old IM patients thus far.

We conducted a systematic review of studies including very old ( $\geq 80$ years) IM patients who underwent a surgical procedure and were evaluated using one or more postoperative outcome measures. The main objective was to report 
short-term (1-month) and 1-year survival rates, as well as performance 1 year after surgery. Our primary hypothesis was that very old IM patients have similar 1-year outcomes as elderly meningioma patients; if so, this may be partly due to a highly specific patient selection process. Since it is likely that reports on very old meningioma patients are retrospective and include a limited number of patients, a descriptive systematic review (rather than meta-analysis) could provide a better overview of the topic.

\section{Materials and methods}

The checklist of the Preferred Reporting Items for Systematic Review and Meta-Analyses (PRISMA) [21] guided our systematic review. A brief description of our research methods is presented below (see Supplementary Material 1 for detailed description).

\section{Study selection}

We used the four-step PICO (patient, intervention, comparison, outcome) principle to frame a study question and eligibility criteria of our systematic literature search [9]. Three different databases, namely Pubmed, Scopus, and Cochrane Library, were used. To be included in the review, studies needed to consist of 80-year-old or older IM patients (patient) who underwent surgical tumor resection (intervention) and to assess postoperative morbidity or mortality (outcome). We excluded commentaries, case reports, case series $(n<5)$, letters, book chapters, reviews, and animal studies. Neither language nor publication year restrictions were used.

\section{Data extraction}

In addition to general demographic characteristics, we extracted information about the following details: study design; indications for surgery; preoperative functional status and comorbidity; size, location, histology, and peritumoral edema of IMs; extent of tumor resection; short-term (1-month) morbidity and mortality; and 1-year morbidity, mortality, and recurrence rates. Furthermore, we tried to review the used prognostic factors and scales for complication-free outcome.

\section{Quality assessment}

According to the Cochrane Collaboration Handbook [8] and Critical Appraisal Skills Program (CASP) [3], we used a domain-based evaluation by six individual domains to assess the quality of each included study: 1) IM characteristics (size, location, histology, and peritumoral edema), 2) preoperative functional status/morbidity, 3) extent of resection, 4) postoperative outcome (morbidity and mortality), and 5) prospective study design. Based on these domains, the studies included were classified as either low, unknown, or high risk of bias. To be classified as a high-quality study, each domain needed to be fulfilled and reported. If one or more domains were missing, we considered that study as high risk of bias, and subsequently classified it as low quality.

\section{Results}

The study selection protocol is depicted in Fig. 1. From a total of 1039 articles, seven fulfilled the inclusion criteria $[5,6,14$, $15,19,20,23]$. All seven studies were retrospective, conducted between 1995 and 2018. Three of the studies were performed in Italy [5, 6, 15], two in France [19, 20], one in Norway [14], and one in the USA [23]. The latter [23] was based on a large national register (Table 1). Altogether, the studies included 308 operated IM patients, varying from 11 to 93 per study. There was an overrepresentation of females in each study (Table 1). One publication [19] was written in French.

\section{Preoperative characteristics}

\section{Preoperative status and morbidity}

All seven studies [5, 6, 14, 15, 19, 20, 23] used the American Society of Anesthesiology (ASA) scale [7] to assess preoperative physical status, which varied considerably among studies. Four studies $[5,6,15,19]$ included mostly healthy patients or patients with mild comorbidities (ASA classes I-II), whereas in three studies [14,20,23], most patients had severe systemic comorbidities (ASA classes III-V). In addition, six studies $[5,6,14,15,19,20]$ used the Karnofsky Performance Scale (KPS) [4] to describe IM patients' preoperative functional status. In these studies, there was an equal representation of dependent (KPS 0-60) and independent (KPS 70-100) IM patients (Table 2). Six studies [5, 6, 14, $19,20]$ reported indications for surgery resection. Motor deficits (27-65\% in three studies), seizures (16-43\% in four studies), and changed mental status (51-59\% in three studies) were the most frequent symptoms leading to resection. Only one study [5] reported surgical treatment outcomes of asymptomatic IM patients $(n=4)$ (Table 2).

\section{Characteristics of IMs}

Three studies $[6,19,20]$ reported complete characteristics (size, location, histology, and peritumoral edema) of IMs. The majority of IMs were WHO grade I (range 64-88\%), located in convexity (range 59-82\%), had moderate or strong peritumoral edema (range 51-100\%), and were categorized as large (at least $4 \mathrm{~cm}$; range 46-81\%) (Fig. 2-d). 
Fig. 1 Study selection protocol

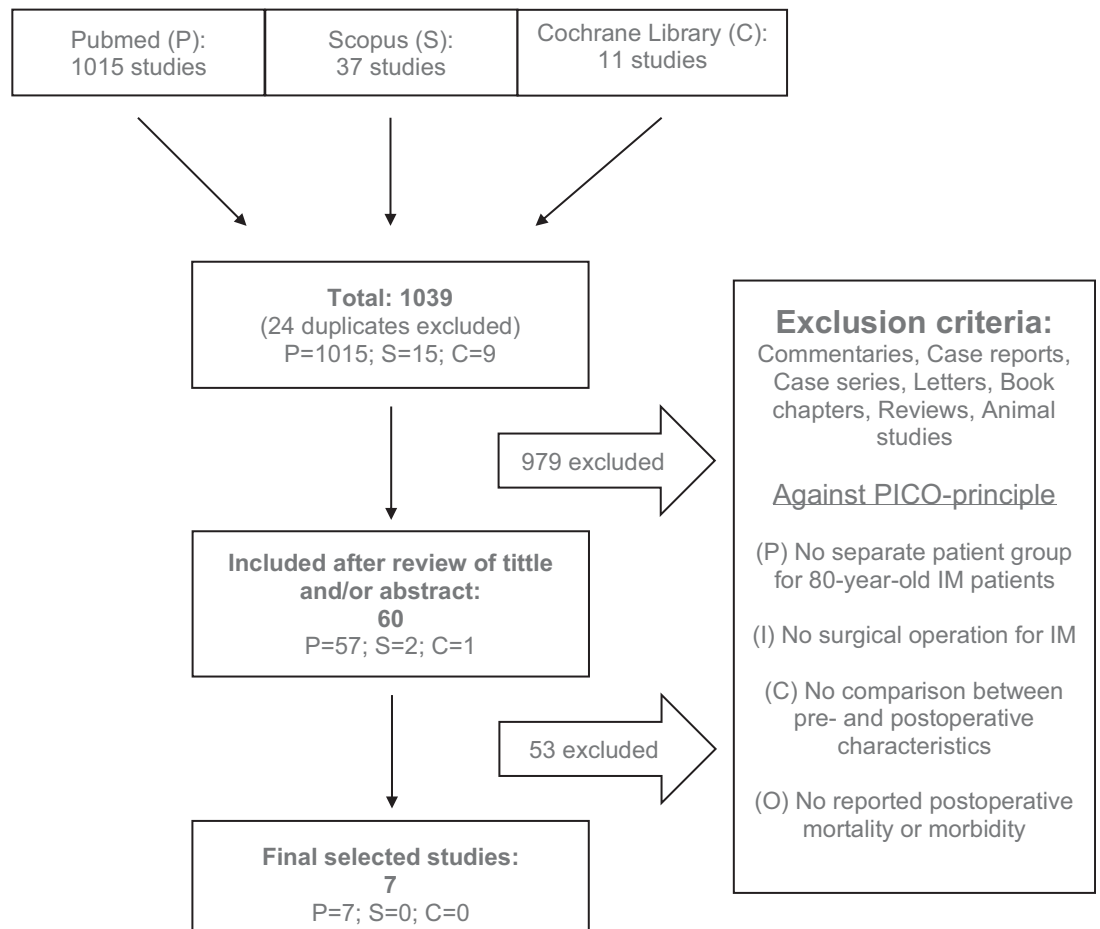

\section{Postoperative characteristics}

\section{Tumor resection}

All except one study [23] reported surgical results (Fig. 3). Total IM resection (Simpson grades I-II) was accomplished with the rate of $72-100 \%$, depending on the study. No case with Simpson grade $\mathrm{V}$ resection (simple decompression with/ without biopsy) was reported.

\section{Postoperative mortality}

All seven studies reported short-term (within 1 month after surgery) mortality rates (Fig. 4). The two earliest studies, partially from the same study population (Mastronardi et al. [15] in 1995, D'Andrea et al. [5] in 2005), reported the highest operative mortality rates of $23.5 \%$ and $13.5 \%$, respectively. In more recent studies, mortality rates were lower $(\leq 8.6 \%)$. Two studies $[19,20]$ did not report short-term mortality. Oneyear mortality rates were reported in four studies $[14,15,19$, 20], with figures ranging from 9.4 to $27.3 \%$. In addition, three studies $[5,15,20]$ reported long-term (over 1-year) follow-up results; for 60- [5], 96- [15], and 147-month [20] follow-ups, the mortality rates were $21.6 \%, 35.3 \%$, and $39 \%$, respectively.

\section{Postoperative morbidity and recurrence}

Overall postoperative complication rates varied between 9.1 and $31.2 \%$, whereas the rate of major complications (death within the first month or a complication requiring reoperation) varied between 0 and $29.4 \%$. The most common major complications were intracerebral hemorrhage (ICH),

Table 1 General characteristics of selected studies

\begin{tabular}{lllllll}
\hline First author, year, and reference & Country & Year & Case number & Age, median/mean (range) & $\%$ of male & Design \\
\hline Mastronardi 1995 [15] & Italy & 1995 & 17 & $82(80-86)$ & 23.5 & Retrospective \\
D’Andrea 2005 [5] & Italy & 2005 & 37 & $82(80-86)$ & 21.6 & Retrospective \\
Riffaud 2007 [19] & France & 2007 & 11 & $82(81-87)$ & 45.5 & Retrospective \\
Sacko 2007 [20] & France & 2007 & 74 & $82^{*}(80-90)$ & 36.5 & Retrospective \\
Konglund 2013 [14] & Norway & 2013 & 51 & $83.4^{*}(80-90)$ & 47.1 & Retrospective \\
Dobran 2018 [6] & Italy & 2018 & 25 & $81.9 *(80-87)$ & 32 & Retrospective \\
Steinberg 2018 [23] & USA & 2018 & 93 & NR (>80) & 37.6 & Retrospective, Register-based \\
\hline
\end{tabular}

$N R$, not reported; *, mean age 
Table 2 Preoperative status and surgery indications

\begin{tabular}{|c|c|c|c|c|c|}
\hline \multirow{3}{*}{$\begin{array}{l}\text { First author, year, and reference } \\
\text { Mastronardi } 1995 \text { [15] }\end{array}$} & \multicolumn{5}{|c|}{ Preoperative status } \\
\hline & \multicolumn{2}{|c|}{ ASA class, $n(\%)$} & \multicolumn{2}{|c|}{ KPS score, $n(\%)$} & \multirow{2}{*}{$\begin{array}{l}\text { Indications of surgery/preoperative symptoms, } n(\%) \\
\text { Impaired mental status: } 10(59)\end{array}$} \\
\hline & I & $2(11.8)$ & $\geq 70$ & $10(59)$ & \\
\hline & II & $11(64.7)$ & $=60$ & $10(59)$ & Asymptomatic: NR \\
\hline & III & $4(23.5)$ & $\leq 50$ & $10(59)$ & \\
\hline & IV & $0(0.0)$ & & & \\
\hline \multirow[t]{10}{*}{ D'Andrea 2005 [5] } & I & $11(33)$ & $\geq 70$ & $23(62)$ & Headache: 27 (73) \\
\hline & II & $19(50)$ & $=60$ & $10(27)$ & Impaired mental status: 19 (51) \\
\hline & III & $7(17)$ & $\leq 50$ & $4(11)$ & Impaired gait: 17 (46) \\
\hline & IV & $0(0.0)$ & & & Seizures: $16(43)$ \\
\hline & & & & & Paresis: $11(30)$ \\
\hline & & & & & Sensory loss: 10 (27) \\
\hline & & & & & Aphasia/dysphasia: 6 (16) \\
\hline & & & & & Visual lost: 5 (13) \\
\hline & & & & & Vertigo: 1 (3) \\
\hline & & & & & Asymptomatic: 4 (11) \\
\hline \multirow[t]{7}{*}{ Riffaud 2007 [19] } & I & $0(0.0)$ & $\geq 80$ & $\geq 80$ & Aphasia/dysphasia: 6 (55) \\
\hline & II & $8(72.7)$ & $\geq 80$ & $\geq 80$ & Impaired mental status: 6 (55) \\
\hline & III & $8(72.7)$ & & & Hemiparesis: 3 (27) \\
\hline & IV & $8(72.7)$ & & & Intracranial hypertension: 1 (9) \\
\hline & & & & & Visual loss: 1 (9) \\
\hline & & & & & Impaired gait: 1 (9) \\
\hline & & & & & Asymptomatic: $0(0)$ \\
\hline \multirow[t]{6}{*}{ Sacko 2007 [20] } & I & $0(0)$ & $\geq 60$ & $42(56.8)$ & Motor deficits: 48 (65) \\
\hline & II & $22(29.7)$ & $\leq 50$ & $32(43.2)$ & Seizures: 32 (43) \\
\hline & III & $44(59.4)$ & & & Intracranial hypertension: 16 (22) \\
\hline & IV & $8(10.8)$ & & & Aphasia/dysphasia: 12 (16) \\
\hline & & & & & Cerebellar symptoms: 12 (16) \\
\hline & & & & & Asymptomatic: $0(0)$ \\
\hline \multirow[t]{3}{*}{ Konglund 2013 [14] } & I-II & $17(33.3)$ & $\geq 80$ & $21(41)$ & Neurological deficits: 29 (54) \\
\hline & III & $30(58.8)$ & $60-70$ & $21(41)$ & Seizures: 22 (43) \\
\hline & IV & $4(7.8)$ & $\leq 50$ & $9(18)$ & Asymptomatic: NR \\
\hline \multirow[t]{4}{*}{ Dobran 2018 [6] } & I & 0 & $\geq 70$ & $21(84)$ & Neurological deficits: 21 (84) \\
\hline & II & $15(60.0)$ & $\geq 70$ & $4(16)$ & Seizures: 4 (16) \\
\hline & III & $10(40.0)$ & & & Asymptomatic: $0(0)$ \\
\hline & IV & 0 & & & \\
\hline \multirow[t]{2}{*}{ Steinberger 2018 [23] } & I-II & $14(15.0)$ & \multirow{2}{*}{\multicolumn{2}{|c|}{ NR }} & NR \\
\hline & III-V & $79(85.0)$ & & & \\
\hline
\end{tabular}

$A S A$, American Society of Anesthesiology scale; KPS, Karnofsky Performance Scale; NR, not reported

cardio-respiratory (CR) failure, and postoperative diffuse edema (PDE) (Table 3). According to five studies [5, 6, 15, 19, 20], the majority $(41.2-86.5 \%)$ of operated IM patients improved their performance (KPS assessments) after the surgery. In addition, when analyzing only the first-year survivors, the proportions of patients with worsened KPS was minimal (0$15.4 \%)$. Four studies $[5,6,15,20]$ reported late recurrence rates, which varied from 0 to $12 \%$. Recurrent meningiomas $(n=4)$ were operated in only one study [20] (Table 3$)$.

\section{Prognostic factors}

Five studies $[5,6,14,15,20]$ reported factors that related to adverse outcome, but only two [14, 20] used adjusted models (Table 4). In multivariate models, several factors including KPS score $\leq 80$, moderate or strong peritumoral edema, male sex, and critical location were related to higher postoperative mortality rates. No predictive factors for postoperative morbidity were found. In univariate models, an ASA class $\geq 3$ was 
Fig. 2 a-d Combined operated IM characteristics of all seven studies

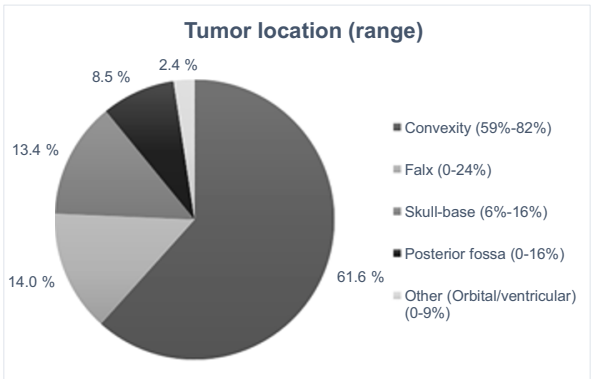

Preoperative peritumoral edema (range)

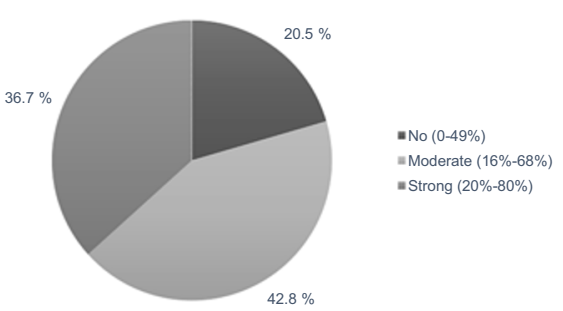

Tumor size (cm) (range)

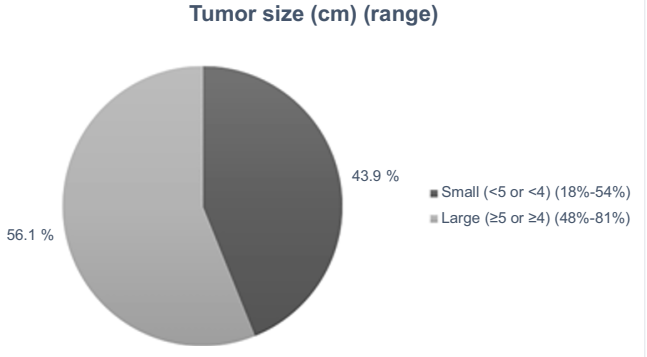

Histology by WHO classification (range)

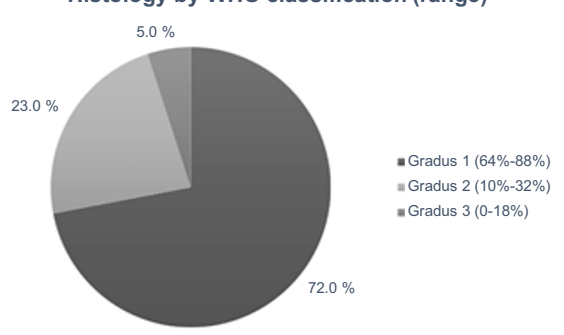

most commonly associated with postoperative mortality, reported in five studies $[5,6,14,15,20]$. For morbidity, four studies $[5,6,15,20]$ reported the relation between an increased tumor size and increased complication rates or postoperative morbidity.

\section{Quality assessment}

The domain-based evaluations are presented in Table 5. None of the included studies fulfilled our criteria for high quality. Major shortcomings and potential sources for various biases include retrospective design (all seven studies), limited reporting of IM characteristics (four studies [5, 14, 15, 23]), and limited outcome assessments (four studies [6, 14, 20, 23]) (Table 5).

\section{Extent of tumor resection (range)}

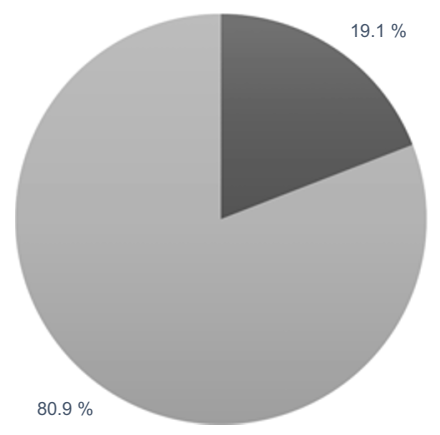

- Partial $(0-28 \%)$

= Total $(72 \%-100 \%)$

Fig. 3 Combined surgery results (\%) of all seven studies

\section{Discussion}

Based on the reviewed literature, seven retrospective studies have assessed surgical outcomes in 80-year-old or older IM patient. In the reviewed studies, 1-month and 1-year mortality varied between $0-23.5 \%$ and $9.4-27.3 \%$, respectively, whereas all five studies $[5,6,15,19,20]$ that reported pre- and postoperative performance levels (ranked by KPS) found that the majority of surgically treated IM patients improved in performance within 6 to 12 months after operation. Based on the available evidence, surgical treatment of IM patients 80 years old or older appears to be a relatively safe procedure, for which the benefits outweigh the potential risks in many patients - especially if preoperative risk assessment and patient selection processes are carefully conducted. Therefore, an increased age should perhaps not be used as a contraindication for meningioma surgery. On the other hand, very old male IM patients with severe comorbidities (ASA $\geq 3$ ), impaired preoperative performance (KPS $\leq 80)$, moderate or

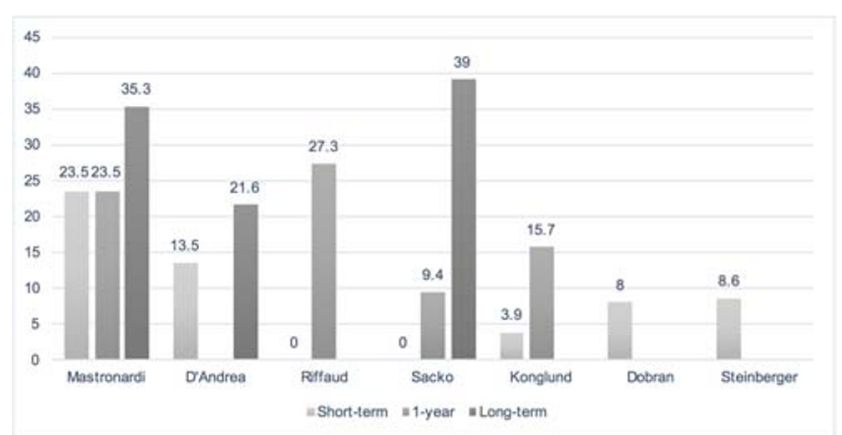

Fig. 4 Short-term (within 30 days), 1-year and long-term (over 1 year) mortality rates $(\%)$ 


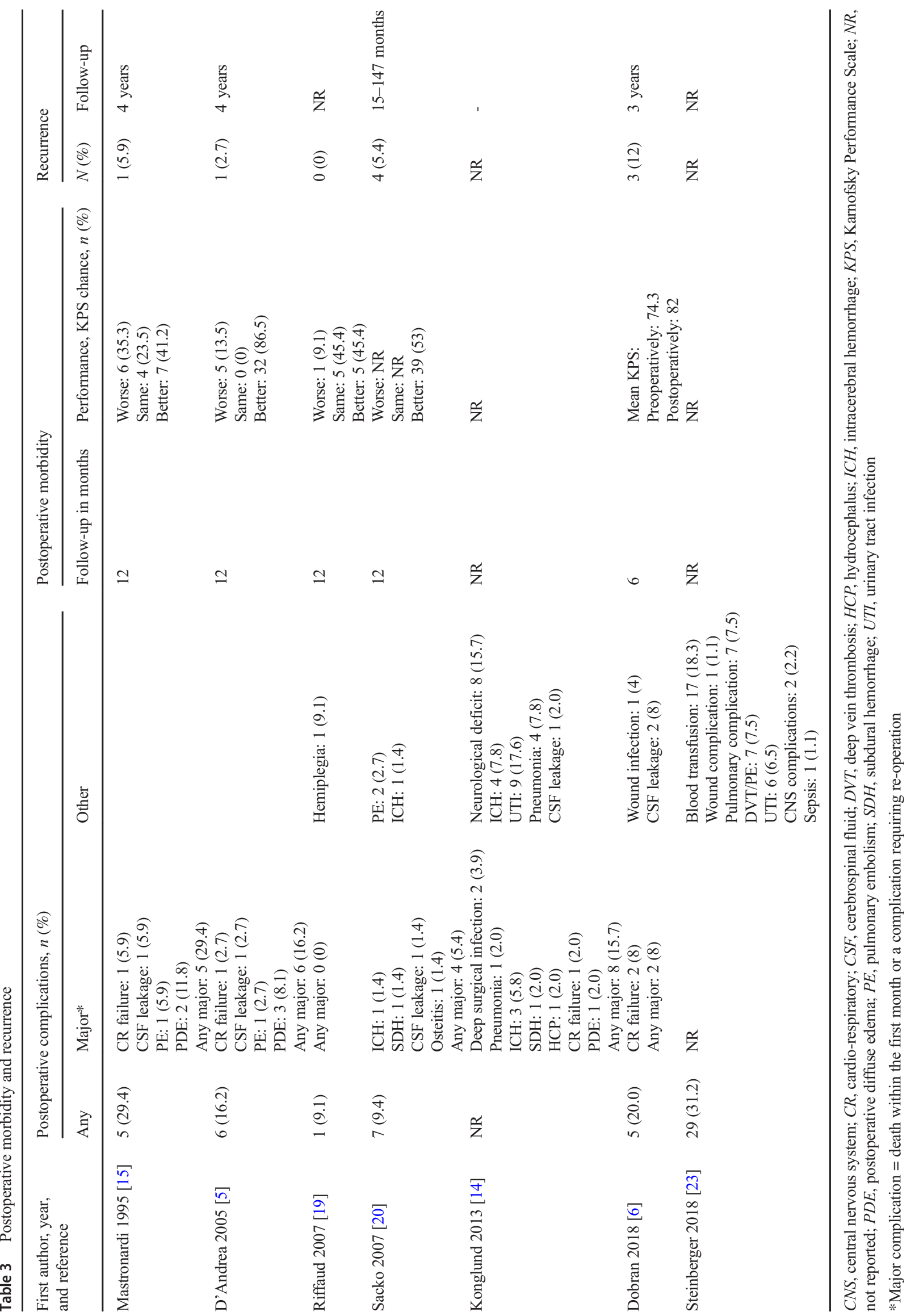


Table 4 Factors related to worse postoperative outcome $(p<0.05)$

\begin{tabular}{|c|c|c|c|c|}
\hline \multirow{3}{*}{$\begin{array}{l}\text { First author, year, } \\
\text { and reference }\end{array}$} & \multicolumn{4}{|l|}{ Prognostic factors } \\
\hline & \multicolumn{2}{|l|}{ For mortality } & \multicolumn{2}{|c|}{ For morbidity or complications } \\
\hline & Univariate & Multivariate & Univariate & Multivariate \\
\hline Mastronardi 1995 [15] & $\begin{array}{l}\text { ASA III } \\
\text { KPS } \leq 60\end{array}$ & NR & $\begin{array}{l}\text { ASA III } \\
\text { Tumor diameter }\end{array}$ & NR \\
\hline D'Andrea 2005 [5] & $\begin{array}{l}\text { ASA III } \\
\text { KPS } \leq 70\end{array}$ & NR & $\begin{array}{l}\text { Tumor diameter } \\
\text { Severe peritumoral edema } \\
\text { Total surgical excision }\end{array}$ & NR \\
\hline Riffaud 2007 [19] & NR & NR & NR & NR \\
\hline Sacko 2007 [20] & $\begin{array}{l}\text { Male sex } \\
\text { KPS } \leq 50 \\
\text { Critical location } \\
\text { ASA III } \\
\text { Severe edema } \\
\text { Low SKALE score }\end{array}$ & Critical location & $\begin{array}{l}\text { Severe edema } \\
\text { Tumor diameter } \\
\text { Total removal }\end{array}$ & None \\
\hline Konglund 2013 [14] & $\begin{array}{l}\text { SKALE }<8 \\
\text { Male sex } \\
\text { ASA } \geq \text { III } \\
\text { Edema }\end{array}$ & $\begin{array}{l}\text { KPS score } \\
\text { Male sex } \\
\text { Edema }\end{array}$ & None & None \\
\hline Dobran 2018 [6] & $\begin{array}{l}\text { Increasing ASA value } \\
\text { Surgical time ( }>240 \mathrm{~min})\end{array}$ & NR & $\begin{array}{l}\text { Tumor diameter }(>4 \mathrm{~cm}) \\
\text { Surgical time }(>240 \mathrm{~min})\end{array}$ & NR \\
\hline Steinberger 2018 [23] & NR & NR & NR & NR \\
\hline
\end{tabular}

ASA, American Society of Anesthesiology scale; KPS, Karnofsky Performance Scale; NR, not reported; SKALE score, sex, KPS, ASA, location, edema score

strong peritumoral edema, and critical tumor location (at the cranial base, near the large vessels, or in eloquent areas) may have a higher risk of postoperative mortality and morbidity.

All seven studies were conducted retrospectively with relatively low case numbers, as hypothesized. According to our quality analysis, shortcomings in IM characterization as well as in comprehensive outcome assessments may lead to a high risk of bias. None of the studies assessed whether the mortality rates were excessive, for example, by comparing the IM patients' postoperative overall survival to the life expectancy of a comparable population. Ideally, the comparison group would contain age- and sex-matched non-operated IM patients with similar comorbidities, but even a comparison to a general population might help to elucidate surgical-related survival risks. Four studies reported 1-year mortality rates: 9.4\% and $27.3 \%$ in France, $29.4 \%$ in Italy, and $15.7 \%$ in Norway. By comparison, current 1-year mortality rates in an age-matched population in these countries are 5.96\% [12], $7.92 \%$ [10], and $8.19 \%$ [22], respectively. This may suggest that surgical treatment is associated with higher mortality in this patient group. However, since the operated patients had often progressive symptoms, which had jeopardized their independency, this comparison to the general population may overestimate the risks of surgery. Nevertheless, the treatment of asymptomatic patients should be carefully considered. The only study [23] that compared the surgical outcome of IM

Table 5 Quality assessment of selected studies. All studies were assigned to the low-quality category. + represents low risk of bias, - high risk of bias, and ? unknown risk of bias

\begin{tabular}{|c|c|c|c|c|c|}
\hline $\begin{array}{l}\text { First author, year, } \\
\text { and reference }\end{array}$ & $\begin{array}{l}\text { IM characteristics } \\
\text { (size, histology, location, edema) }\end{array}$ & $\begin{array}{l}\text { Preoperative } \\
\text { morbidity }\end{array}$ & $\begin{array}{l}\text { Extent of } \\
\text { resection }\end{array}$ & $\begin{array}{l}\text { Comprehensive outcome } \\
\text { assessment (morbidity and mortality) }\end{array}$ & Prospective design \\
\hline Mastronardi 1995 [15] & - & + & + & + & - \\
\hline D'Andrea 2005 [5] & - & + & + & + & - \\
\hline Riffaud 2007 [19] & + & + & + & + & - \\
\hline Sacko 2007 [20] & + & + & + & - & - \\
\hline Konglund 2013 [14] & $?$ & + & + & - & - \\
\hline Dobran 2018 [6] & + & + & + & - & - \\
\hline Steinberger 2018 [23] & - & + & - & - & - \\
\hline
\end{tabular}

$I M$, intracranial meningioma 
patients under 80 years old with those over 80 years old reported that age over 80 years was an independent risk factor for postoperative complications $(\mathrm{OR}=2.4 ; 95 \%$ confidence interval (CI) 1.3-4.4)) and short-term mortality $(\mathrm{OR}=15.7$; CI 3.0-81.0). Thus, always aiming at radical removal at the expense of higher complication rates may not provide the best possible outcome, especially in this age group, since the growth rate of meningiomas is ultimately slow. In terms of functional recovery, five studies used KPS as a postoperative outcome assessment, but future studies could also consider simpler outcome measures such as ability to live at home after the surgery. This could perhaps provide more relevant information about IM patients' dependency, recovery, and performance status.

Previously, two systematic reviews have examined surgical outcomes in elderly ( 65 years or older) IM patients. In 2017, the review by Ikawa et al. [11] included three studies [5, 14, $20]$ with very old (80-year-old or older) IM patients; in 2014, the review by Poon et al. [17] included two such studies [5, $20]$. Despite the fact that these two reviews also included younger populations, their reported short-term $(0-10.8 \%$ and $0-12 \%)$ and 1 -year mortality rates $(0-16.7 \%$ and $6.3-15.6 \%)$ were very similar to our findings. The same was true for complication rates (2.7-49.4\% and 2.7-29.8\%) and prognostic factors (ASA class, KPS, and peritumoral edema).

Due to the increased life expectancy, improved diagnostic modalities, and increased treatment options, the incidence of IMs in older populations has increased during recent decades [16]. Presently, the incidence of IMs in the general population is about one in every 12,500 people [16]. However, as the incidence rises with increasing age, the rate of occurrence increases to roughly one in 2000 [16] among very old (80year-old and older) individuals - nearly twice as high as in 70year-olds, and over five times higher than in 50-year-olds [16]. The physical condition and independence of these very old IM patients continue to improve, and these patients may retain their activity up to 90-95 years old. Therefore, this patient group needs to be studied in greater detail, especially in terms of the safety and benefits of surgical treatment.

While our review provides important insights into this topic, it is not without limitations. First, even though we performed our systematic literature search using three different databases, we may have missed some relevant studies. Second, due to the descriptive nature of the reviewed studies and the high risk of biases, we used only qualitative analyses to describe the current scientific evidence. Third, due to retrospective design of all reviewed studies, we believe that future studies with prospective data collection may provide more reliable information about the postoperative complications and their clinical impact on this patient group. Nevertheless, this systematic review provides the first overview of the scientific evidence for surgical treatment of 80-year-old or older IM patients and also guides future studies to avoid the critical shortcomings presented in the review. In addition, we believe that our review may aid in critical surgical decision-making processes.

\section{Conclusion}

After a careful patient selection process, surgical removal of IM appears to be a relatively safe procedure, even in 80-yearold and older meningioma patients. However, prospective studies should confirm these findings by comparing postoperative outcomes, ideally to a matched IM patient group that undergo conservative treatment.

Acknowledgments We would like to thank Jacquelin DeFaveri for language revision. IR would like to thank Maire Taponen and Kunnanlääkäri Uulo Arhio Foundations for research grants.

Author contributions All authors contributed to the study conception and design. Literature search and data analysis were performed by IR. The first draft of the manuscript was written by IR and all authors commented on previous versions of the manuscript. All authors read and approved the final manuscript.

Funding information Open access funding provided by University of Helsinki including Helsinki University Hospital. IR received research grants from Maire Taponen and Kunnanlääkäri Uulo Arhio foundations. Reported foundation has no personal or institutional financial interests concerning this study.

\section{Compliance with ethical standards}

Conflict of interest The authors declare that they have no conflict of interest.

Ethical approval This article does not contain any studies with human participants or animals performed by any of the authors.

Informed consent The review was based on systematic literature search and thus, included no personal data.

Open Access This article is licensed under a Creative Commons Attribution 4.0 International License, which permits use, sharing, adaptation, distribution and reproduction in any medium or format, as long as you give appropriate credit to the original author(s) and the source, provide a link to the Creative Commons licence, and indicate if changes were made. The images or other third party material in this article are included in the article's Creative Commons licence, unless indicated otherwise in a credit line to the material. If material is not included in the article's Creative Commons licence and your intended use is not permitted by statutory regulation or exceeds the permitted use, you will need to obtain permission directly from the copyright holder. To view a copy of this licence, visit http://creativecommons.org/licenses/by/4.0/.

\section{References}

1. Ambekar S, Sharma M, Madhugiri VS, Nanda A (2013) Trends in intracranial meningioma surgery and outcome: a Nationwide 
Inpatient Sample database analysis from 2001 to 2010. J NeuroOncol 114:299-307. https://doi.org/10.1007/s11060-013-1183-6

2. Cahill KS, Claus EB (2011) Treatment and survival of patients with nonmalignant intracranial meningioma: results from the surveillance, epidemiology, and end results program of the National Cancer Institute. Clinical article J Neurosurg 115:259-267. https:// doi.org/10.3171/2011.3.JNS101748

3. Critical Appraisal Skills Programme (CASP) (2014) CASP Checklists (http://media.wix.com/ugd/dded87_ e37a4ab637fe46a0869f9f977dacf134.pdf)

4. Crooks V, Waller S, Smith T, Hahn TJ (1991) The use of the Karnofsky Performance Scale in determining outcomes and risk in geriatric outpatients. J Gerontol 46:M139-M144

5. D'Andrea G, Roperto R, Caroli E, Crispo F, Ferrante L (2005) Thirty-seven cases of intracranial meningiomas in the ninth decade of life: our experience and review of the literature. Neurosurgery 56:956-961 discussion 956-961

6. Dobran M, Marini A, Nasi D, Liverotti V, Benigni R, Iacoangeli M, Scerrati M (2018) Surgical treatment and outcome in patients over 80 years old with intracranial meningioma. Clin Neurol Neurosurg 167:173-176. https://doi.org/10.1016/j.clineuro.2018.02.024

7. Doyle Dj Fau - Garmon EH, Garmon EH (2019) American Society of Anesthesiologists Classification (ASA Class) BTI - StatPearls

8. Higgins JPT GS (2011) Cochrane handbook for systematic reviews of interventions version 5.1.0 [updated March 2011]

9. Huang X, Lin J, Demner-Fushman D (2006) Evaluation of PICO as a knowledge representation for clinical questions. AMIA Annu Symp Proc:359-363

10. I.stat (2017) Deaths of resident population (http://dati.istat.it/Index. aspx? DataSetCode=DCIS_MORTALITA1\&Lang=en)

11. Ikawa F, Kinoshita Y, Takeda M, Saito T, Yamaguchi S, Yamasaki F, Iida K, Sugiyama K, Arita K, Kurisu K (2017) Review of current evidence regarding surgery in elderly patients with meningioma. Neurol Med Chir 57:521-533. https://doi.org/10.2176/nmc.ra. 2017-0011

12. INED (2017) Mortality rates by sex and age in 2016 (https://www. ined.fr/en/everything_about_population/data/france/deaths-causesmortality/mortality-rates-sex-age/)

13. Kleihues P, Louis DN, Scheithauer BW, Rorke LB, Reifenberger G, Burger PC, Cavenee WK (2002) The WHO classification of tumors of the nervous system. J Neuropathol Exp Neurol 61:215-225; discussion 226-219. https://doi.org/10.1093/jnen/61.3.215

14. Konglund A, Rogne Sg Fau - Helseth E, Helseth E Fau - Meling TR, Meling TR (2013) Meningioma surgery in the very oldvalidating prognostic scoring systems

15. Mastronardi L, Ferrante L, Qasho R, Ferrari V, Tatarelli R, Fortuna A (1995) Intracranial meningiomas in the 9th decade of life: a retrospective study of 17 surgical cases. Neurosurgery 36:270 274. https://doi.org/10.1227/00006123-199502000-00005
16. Ostrom QT, Gittleman H, Truitt G, Boscia A, Kruchko C, Barnholtz-Sloan JS (2018) CBTRUS statistical report: primary brain and other central nervous system tumors diagnosed in the United States in 2011-2015. Neuro Oncol 20:iv1-iv86. doi:https:// doi.org/10.1093/neuonc/noy131

17. Poon MT, Fung LH, Pu JK, Leung GK (2014) Outcome of elderly patients undergoing intracranial meningioma resection - a systematic review and meta-analysis. Br J Neurosurg 28:303-309. https:// doi.org/10.3109/02688697.2013.841857

18. Reponen E, Korja M, Niemi T, Silvasti-Lundell M, Hernesniemi J, Tuominen H (2015) Preoperative identification of neurosurgery patients with a high risk of in-hospital complications: a prospective cohort of 418 consecutive elective craniotomy patients. J Neurosurg 123:594-604. https://doi.org/10.3171/2014.11.JNS141970

19. Riffaud L, Mazzon A Fau - Haegelen C, Haegelen C Fau - Hamlat A, Hamlat A Fau - Morandi X, Morandi X (2007) Surgery for intracranial meningiomas in patients older than 80 years (Chirurgie des meningiomes intracraniens apres 80 ans. DEP 20061212)

20. Sacko O, Sesay M, Roux FE, Riem T, Grenier B, Liguoro D, Loiseau H (2007) Intracranial meningioma surgery in the ninth decade of life. Neurosurgery 61:950-954; discussion 955. https:// doi.org/10.1227/01.neu.0000303190.80049.7d

21. Shamseer L, Moher D, Clarke M, Ghersi D, Liberati A, Petticrew M, Shekelle P, Stewart LA, Group P-P (2015) Preferred reporting items for systematic review and meta-analysis protocols (PRISMAP) 2015: elaboration and explanation. BMJ 350:g7647. https://doi. org/10.1136/bmj.g7647

22. Statistics-Norway (2018) Age-specific death rates for males and females (https://www.ssb.no/en/befolkning/statistikker/dode)

23. Steinberger J, Bronheim RS, Vempati P, Oermann EK, Ladner TR, Lee NJ, Kothari P, Caridi JM, Shrivastava RK (2018) Morbidity and mortality of meningioma resection increases in octogenarians. World Neurosurg 109:e16-e23. https://doi.org/10.1016/j.wneu. 2017.09.021

24. United Nations, Department of Economic and Social Affairs, Population Division (2017). World Population Prospects: The 2017 Revision

25. Wiemels J, Wrensch M, Claus EB (2010) Epidemiology and etiology of meningioma. J Neuro-Oncol 99:307-314. https://doi.org/10. 1007/s11060-010-0386-3

26. Wilmoth JR (2000) Demography of longevity: past, present, and future trends. Exp Gerontol 35:1111-1129

Publisher's note Springer Nature remains neutral with regard to jurisdictional claims in published maps and institutional affiliations. 